\title{
Vaginal Hemorrhage, CTCAE
}

National Cancer Institute

\section{Source}

National Cancer Institute. Vaginal Hemorrhage, CT CAE. NCI Thesaurus. Code C56573.

A disorder characterized by bleeding from the vagina. 